\title{
Determination of neutron temperature in irradiation channels of reactor
}

\author{
Tran Van Hung
}

Received: 30 May 2010/Published online: 13 July 2010

(C) The Author(s) 2010. This article is published with open access at Springerlink.com

\begin{abstract}
The neutron temperature is a characteristic parameter in irradiation channels of reactor. For nuclides which have resonances in the thermal neutron energy range, their Westcott g-factors are different from unity. The values of g-factors and cross-sections of $(\mathrm{n}, \gamma)$ reaction of these nuclides are temperature dependence. The standard energy for tabulation of thermal neutron cross-section $\left(\sigma_{0}\right)$ is that of room temperature $\left(293.59 \mathrm{~K}\right.$ or $\left.20.43{ }^{\circ} \mathrm{C}\right)$, corresponding to a neutron energy $0.0253 \mathrm{eV}$ or to a neutron velocity of $2200 \mathrm{~m} / \mathrm{s}$. However, in the irradiation channels of reactor, the temperature is not exact at $20.43{ }^{\circ} \mathrm{C}$. Thus, the temperature at the irradiation position must be known to convert $\sigma_{0}$ to $\sigma(\mathrm{T})$. A method for determination of the neutron temperature in irradiation channels of Dalat reactor is presented by fitting the thermal neutron spectrum obtained from the calculation using MCNP code.
\end{abstract}

Keywords Westcott g-factor - Neutron temperature . Dalat reactor $\cdot$ MCNP code

\section{Introduction}

In the activation measurements using the nuclear reactor, the neutron capture reaction rates are the product of the neutron flux at the sample position and the neutron capture cross-section of irradiation nuclide. The neutron capture cross-section at particular neutron energy is the probability that a neutron at that energy will be captured by the

T. Van Hung $(\bowtie)$

Research and Development Center for Radiation Technology, 202A, 11 Str, Linh Xuan, Thu Duc, Ho Chi Minh, Vietnam

e-mail: tranhungkeiko@yahoo.com nuclide. The standard energy for tabulation of thermal neutron cross-section $\left(\sigma_{0}\right)$ is that of room temperature $\left(293.6^{\circ} \mathrm{K}\right.$ or $20.43{ }^{\circ} \mathrm{C}$ ), corresponding to a neutron energy $0.0253 \mathrm{eV}$ or to a neutron velocity of $2200 \mathrm{~m} / \mathrm{s}$. However, in many reactors, the temperature $(\mathrm{T})$ in the irradiation channels is not $20.43{ }^{\circ} \mathrm{C}$, but at higher temperature. Westcott developed a method for converting $\sigma_{0}$ to $\sigma(\mathrm{T})$, which is the effective cross-section at the actual temperature $(\mathrm{T})$ of the irradiation channel, by describing the neutron spectrum as a combination of a Maxwellian distribution function, which is characterized by a temperature (T), and a component of epithermal energy neutrons [1].

According to Westcott, the reaction rate per target atom is given by:

$R=\phi_{0} \stackrel{\Lambda}{\sigma}(T)=n v_{0} \sigma_{0}\left[g(T)+r \sqrt{T / T_{0}} s_{0}\right]$

where $\phi_{0}=n v_{0}$ neutron flux defined as the total neutron density times the $2200 \mathrm{~m} / \mathrm{s}$ neutron velocity; $\stackrel{\Lambda}{\sigma}(T)$ effective cross-section; $\sigma_{0}$ thermal cross-section for $2200 \mathrm{~m} / \mathrm{s}$ neutron; $g(T)$ parameter which represents the departure of the cross-section from the $1 / \mathrm{v}$ law in the thermal region $(g(T)=1$ if nuclide obeys the $1 / \mathrm{v}$ law in this energy region) and which can be calculated from expression:

$g(T)=\frac{2}{\sqrt{\pi E_{0}} \sigma_{0}} \int_{0}^{\infty} \sqrt{E} \sigma(E) \sqrt{E / E_{T}} e^{-E / E_{T}} d E / E_{T}$

where $E_{T}=E_{0} T / T_{0}, E_{0}=0.0253 \mathrm{eV}, T_{0}=293.6^{\circ} \mathrm{K}$, $E$-neutron energy, and v-neutron velocity. The $g(T)$-factors with the temperatures from $273.16 \mathrm{~K}\left(0{ }^{\circ} \mathrm{C}\right)$ to $673.16 \mathrm{~K}$ $\left(400{ }^{\circ} \mathrm{C}\right)$ for many nuclides were calculated by Holden [2].

$r \sqrt{T / T_{0}}$ - epithermal index which denotes the strength of the epithermal flux (it is zero for a pure thermal flux) and 
$s_{0}$ - parameter which represents the ratio of the resonance integral and thermal cross-section

According to the Hogdahl's convention [3], a method, theoretically less rigorous, but commonly used defines the reaction rate by:

$R=\phi_{t h} \phi_{t h}+\phi_{e p i} I$

where $\sigma_{t h}, I$ sub-cadmium cross-section and the epi-cadmium cross-section, respectively; $\phi_{t h}$ neutron flux defined as the thermal neutron density times the $2200 \mathrm{~m} / \mathrm{s}$ neutron velocity; $\phi_{e p i}$ epithermal flux per unit $\ln (\mathrm{E})$.

From Eqs. 1 and 3 it follows that (see [4]):

$\sigma_{t h}=g(T) \sigma_{0}+\frac{\phi_{e p i}}{\phi_{t h}}\left(1-2 \sqrt{E_{0}-E_{c}}\right) g(T) \sigma_{0}$

or

$\sigma_{t h}=g(T) \sigma_{0}+\frac{\phi_{e p i}}{\phi_{t h}}\left[\Delta I^{\prime}-I(1 / v)\right]$

with

$I(1 / v)=\int_{E_{C}}^{\infty} g(T) \sigma_{0} \sqrt{E_{0} / E} d E / E \cong 2 g(T) \sigma_{0} \sqrt{E_{0} / E_{c}}$

where $E_{c}$-Cd-cut-off energy (this cut-off energy is a function of thickness of $\mathrm{Cd} ; E_{c}=0.55 \mathrm{eV}$ if a small cylindrical Cd-filter of $1 \mathrm{~mm}$ thickness), $I(1 / v)-1 / \mathrm{v}$-part of resonance integral, $I^{\prime}$ and $\Delta I^{\prime}$ is the epi-cadmium resonance integral excluding the $1 / \mathrm{v}$ part and part, shielded by Cd-filter, which depends on the neutron temperature, respectively.

Clearly, looking at Eqs. 1, 3, 4, 5, and 6 there is one notice that the cross-sections and the resonance integral vary as a function of $\mathrm{g}(\mathrm{T})$, which are temperature dependence. Many authors often assume $\sigma_{t h}=\sigma_{0}$ to calculate the resonance integral, it is not completely justified. Only the nuclides with cross-section obey the $1 / \mathrm{v}$ law up to $1-2 \mathrm{eV}, \mathrm{g}(\mathrm{T})$ would be unity as mentioned above and $\mathrm{s}_{0}=0$; then $\sigma_{t h}$ and the resonance integral are no longer depends on temperature at the irradiation position. Thus, in the activation measurements using the nuclear reactor, neutron temperature $(\mathrm{T})$ at the irradiation position should be known to preserve the accuracy of the results.

In experiment, a well-known method for the determination of the neutron temperature in the irradiation channels is co-irradiation of $\mathrm{Lu}$ by using ${ }^{176} \mathrm{Lu}(\mathrm{n}, \gamma){ }^{177} \mathrm{Lu}$ reaction and $1 / \mathrm{v}$-monitors [5] with acceptable accuracy. In this work, we describe a method for the determination of the neutron temperature based on thermal neutron spectrum calculated by MCNP code.

\section{Base of method}

As we know, neutron spectrum at an irradiation position can be expressed as a sum of a thermal equilibrium spectrum; $\varphi_{\mathrm{th}}(\mathrm{E})$ (Maxwellian distribution, neutron energy from 0 to $0.55 \mathrm{eV}$ ) and epithermal spectrum in the slowing down region; $\varphi_{\text {epi }}(\mathrm{E})$ (neutron energy from $0.55 \mathrm{eV}$ to maximal). In the paper $[6,7]$, the neutron spectra were written by semi-empirical functions:

$\varphi_{t h}(E)=I_{t h}\left(\frac{E}{E_{T}^{2}}\right) e^{-\frac{E}{E_{T}}}$

$\varphi_{\text {epi }}(E)=I_{\text {epi }}\left(\frac{E}{1 e V}\right)^{-\alpha} \cdot \frac{1}{E}$

where $I_{t h}$ and $I_{\text {epi }}$ are scaling constants for the thermal and epithermal portion; $E_{T}$ is a characteristic energy of Maxwellian portion of the spectrum. The neutron temperature is given by:

$E_{T}(e V)=8.617 \times 10^{-5} T$

$\mathrm{T}$ is in degrees $\mathrm{K}$ (degrees Kelvin). From the temperature in degrees Kelvin, we can convert to temperature in degrees Celsius by: T-273.15.

Thus, if the neutron spectrum at the irradiation position is known, $E_{T}$ would be determined by fitting Maxwellian portion of the spectrum according to Eq. 7. From energy $E_{T}$, neutron temperature $\mathrm{T}$ would be also calculated by using Eq. 9. This method was applied to determinate neutron temperature in the irradiation channels 7-1, 1-4, the rotary specimen rack and neutron trap of Dalat reactor, Vietnam.

\section{MCNP code and configuration of Dalat reactor core}

\section{MCNP code}

MCNP code was developed by the Los Almos National Laboratory. It is a general purpose Monte Carlo code [8], which facilitates independent or coupled neutron, photon and electron transport calculations. The code treats an arbitrary three-dimensional configuration of material and geometric cell and provides a versatile description of the source, the variance reduction techniques, a flexible tally structure and an extensive collection of cross-section data in continuous energy representation. For neutron, all reactions given in a particular cross-section data evaluation are accounted for and cover the energy range from $10^{-11}$ to $20 \mathrm{MeV}$.

Jacimovíc et al. [9] used MCNP version 4B for the calculation of spectral parameters in typical irradiation channels of the TRIGA reactor, Ljubljuna. The comparison 
between the simulated and experimentally determined parameter $\mathrm{f}$ is in good agreement except for channel IC40. Hung et al. [6, 7] also used MCNP for calculation of some characteristic parameters of neutron flux in irradiation channels of Dalat reactor. The comparison of results obtained by experimental and Monte Carlo simulation showed good agreement for all channels. The obtained results in that papers showed that Monte Carlo calculation of neutron spectra in irradiation channels of reactor provide useful information about the nature of spectra, the neutron flux, as well as reactor's spectral characteristics.

\section{Configuration of Dalat reactor core}

The Dalat reactor is a pool type research reactor. Distilled water is used as moderator, coolant, reflector and biological protection. The cylindrical reactor is arranged symmetrically by several kinds of material elements. In the center of active region is a neutron trap of radius $3.2 \mathrm{~cm}$. The fuel elements, beryllium and graphite blocks, control rods and irradiation channels are the active region of reactor and form a hexagonal prism lattice in the cylindrical reactor with radius $20.8 \mathrm{~cm}$ and height $60.0 \mathrm{~cm}$. Outside of the active region is a graphite reflector with thickness of $32.6 \mathrm{~cm}$. The working configuration of reactor core is shown in Fig. 1.

The detailed MCNP computation model used for the calculation of neutron spectra in irradiation channels of $7-1,1-4$, the rotary specimen rack and neutron trap of Dalat reactor is based on a model thoroughly described by Hung et al. [6, 7]. In the present problem, MCNP code version 4C is used. KCODE (criticality problem) and KSRC (initial spatial distribution of source points) cards are used for the source description. In this case, MCNP offers several options for the $\mathrm{k}_{\mathrm{eff}}$ values estimate, including the combined average of the absorption, collision and track-length estimator.

For all calculations of neutron spectra, the F4 tally was used. F4 is a track-length tally card for calculating average neutron flux over a cell. Nuclide cross-sections were taken from JENDL-3.2 [10]. In the calculation, the $S(\alpha, \beta)$ thermal treatment was used for $\mathrm{H}_{2} \mathrm{O}$, beryllium and graphite.

\section{Results and discussion}

The calculated $\mathrm{k}_{\text {eff }}$ value was $1.0010 \pm 0.0005$. The calculation simulated a critical state. Therefore, this value is quite satisfactory. The energy spectra with 115 groups of energy from $0 \mathrm{eV}$ to $20 \mathrm{MeV}$ at 1-4, 7-1 channels and rotary specimen rack and neutron trap are calculated. As an example, Fig. 2 shows a calculated energy spectrum in 7-1
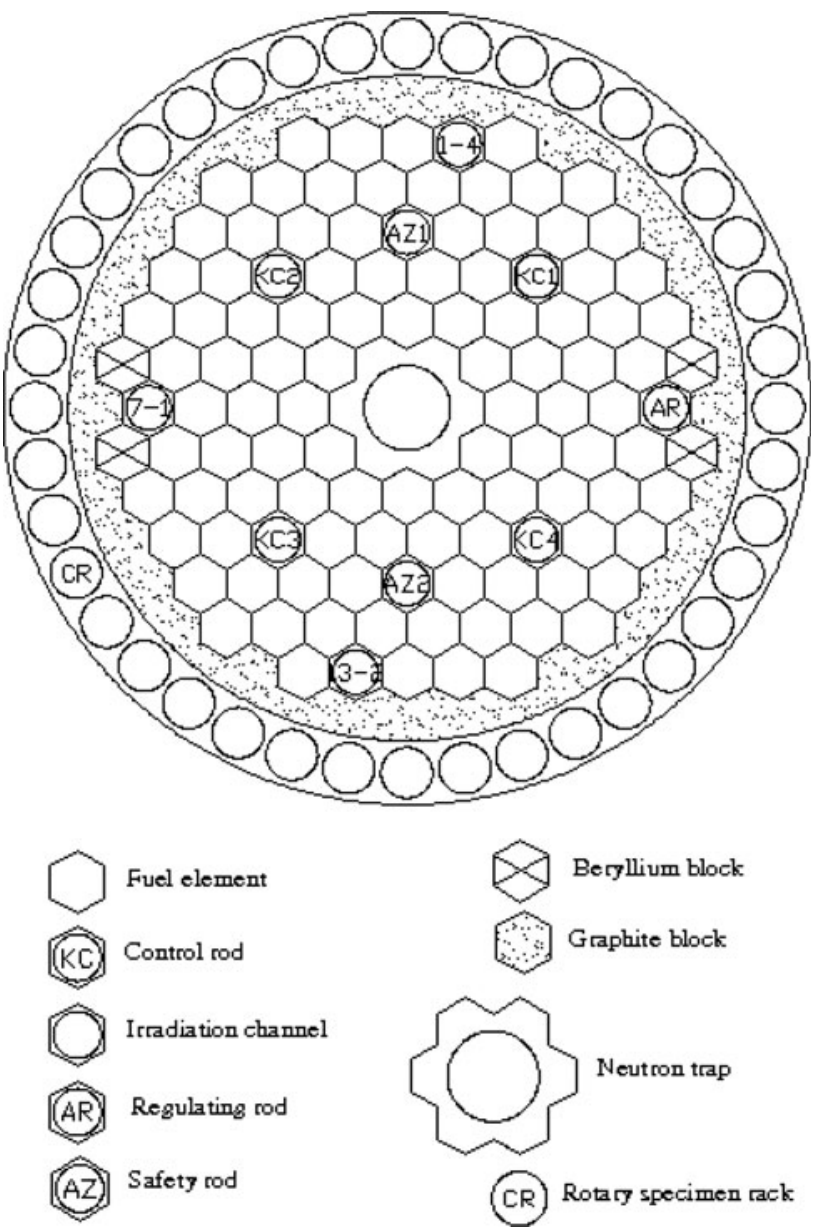

Fig. 1 Configuration of reactor core of Dalat reactor

channel and the fitted $\mathrm{E}_{\mathrm{T}}$-value in the thermal neutron region. The relative error of the calculated spectrum

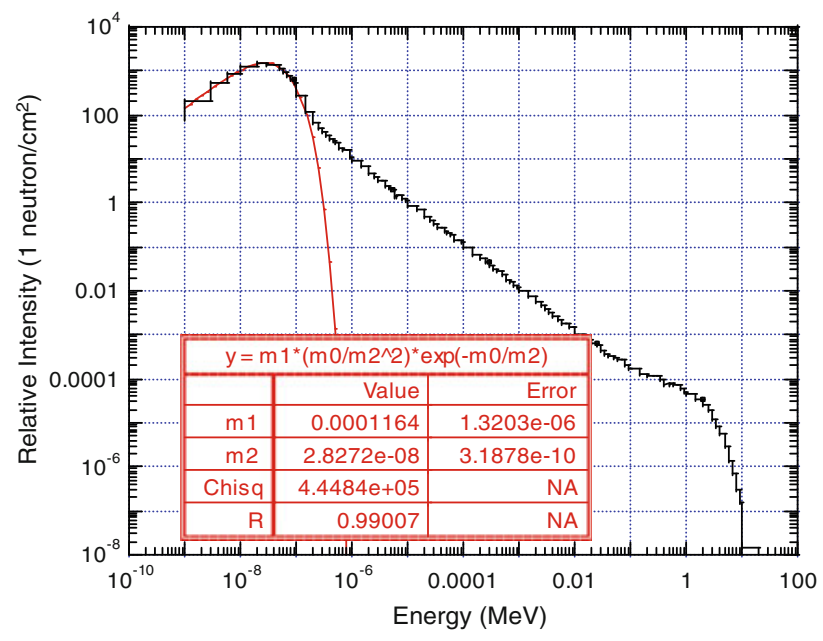

Fig. 2 Calculated neutron flux spectrum in 7-1 channel of Dalat reactor using $\mathrm{MCNP} 4 \mathrm{C}$ code (dark line) and fitted $\mathrm{E}_{\mathrm{T}}$-value in the thermal neutron region (light line) 
Table 1 The fitted $\mathrm{E}_{\mathrm{T}}$ values and the neutron temperatures in irradiation channels of Dalat reactor determined from the neutron spectra calculated by MCNP code and experiment

\begin{tabular}{llll}
\hline Irradiation channels & $\begin{array}{l}\text { Fitted } \mathrm{E}_{\mathrm{T}^{-}} \text {values } \\
\left(10^{-2} \mathrm{eV}\right)\end{array}$ & $\begin{array}{l}\text { Calculated } \\
\text { temperatures }\left({ }^{\circ} \mathrm{C}\right)\end{array}$ & $\begin{array}{l}\text { Experimental } \\
\text { temperatures }\left({ }^{\circ} \mathrm{C}\right) \\
\text { Lu-Au monitor }\end{array}$ \\
\hline Neutron trap & $2.714 \pm 0.028$ & $41.8 \pm 1.0$ & \\
1-4 channel & $2.727 \pm 0.029$ & $43.3 \pm 1.0$ & $53.0 \pm 2.0$ \\
7-1 channel & $2.827 \pm 0.031$ & $52.0 \pm 1.0$ & \\
Rotary specimen rack & $2.698 \pm 0.027$ & $40.0 \pm 1.0$ & \\
\hline
\end{tabular}

intensity in thermal neutron region is about than $1 \%$ and in the epithermal is about 3\% for all irradiation channels.

From the neutron spectra calculated by MCNP code, the $\mathrm{E}_{\mathrm{T}}$ values are determined by fitting the neutron spectra in the thermal neutron region (neutron energy from 0 to $0.55 \mathrm{eV}$ ) according to Eq. (7) using Kaleigraph program. The $\mathrm{E}_{\mathrm{T}}$-fitting values and the temperatures in degrees Celsius in the irradiation channels are presented in Table 1. The uncertainty of the neutron temperature values determined from fitting the calculated energy spectra in the thermal neutron region is about 2.5\%; it defends on the uncertainty of the calculated spectrum intensity in thermal neutron region.

As a comparison to fitted value, an experiment of the T-determination in 7-1 channel was performed. The choice of 7-1 channel is suitable for the experiment due to 7-1 channel coupled with a pneumatic sample transfer system. The neutron temperature is determined by co-irradiating a $\mathrm{Lu}-\mathrm{Au}$ monitor. The T-determination method was presented in paper [4]. After measuring the irradiated monitors, the WESTCOTT factor for lutetium $[\mathrm{g}(\mathrm{T})]_{\mathrm{Lu}}$ could be obtained. Thus, the neutron temperature also was determined by using the tables $[\mathrm{g}(\mathrm{T})]_{\mathrm{Lu}}$ vs. $\mathrm{T}_{\mathrm{n}}$ in paper [2]. The comparison of the result of the neutron temperature in 7-1 channel shows that the value of the neutron temperature agrees well with each other. The difference between both data is about $2 \%$.

From Table 1, it also shows that the neutron temperatures in the irradiation channels of Dalat reactor are from $40{ }^{\circ} \mathrm{C}$ (in the rotary specimen rack) to $53{ }^{\circ} \mathrm{C}$ (7-1 channel) in the core of reactor. The neutron temperature in 7-1 channel is highest, because it is a dry channel using for neutron activation analysis (NAA) only.

Note that, in general, the recommended $\mathrm{k}_{0, \mathrm{Au}}$ using in the neutron activation analysis in reports is the average $\mathrm{k}_{0, \mathrm{Au}^{-}}$-value corresponding to an average neutron temperature of $60{ }^{\circ} \mathrm{C}$ [11]. Hence, in $\mathrm{k}_{0}$ NAA standardization method, the neutron temperature at the irradiation position is importance for nuclides having $(n, \gamma)$ reaction which do not exactly obeys the $\sigma(\mathrm{v}) \sim 1 / \mathrm{v}$ law up to $1-2 \mathrm{eV}$. The cross-section of $(n, \gamma)$ reaction of these nuclides depends clearly on the neutron temperature. For this reason, 7-1 channel is suitable in NAA using $\mathrm{k}_{0}$ standardization method, because there is no need of the correction of neutron temperature.

\section{Conclusion}

The neutron temperature values in the channels of 7-1, 1-4, the rotary specimen rack and neutron trap of Dalat reactor were determined from the neutron spectra in the thermal neutron region, which was calculated by MCNP code. It was compared with the experimental value in 7-1 channel and showed that the values are agreement with each other. The difference between both data is about $2 \%$.

The results from this study suggest that base on the neutron spectrum calculated by MCNP code, the neutron temperature in the irradiation channels of reactor will be determined by fitting the spectrum in the thermal neutron region according to Eq. 7. Using this method, it is simple, effective and is a good method for the verification of experimental results.

Open Access This article is distributed under the terms of the Creative Commons Attribution Noncommercial License which permits any noncommercial use, distribution, and reproduction in any medium, provided the original author(s) and source are credited.

\section{References}

1. Westcott CH, Walker WH, Alexander TK (1958) In: 2nd International conference paceful uses of atomic energy. IAEA, Geneva 16:70

2. Holden NE (1999) Pure Appl Chem 71:2309

3. HØdahl OT (1962) Report MMPP-226-1, University of Michigan, Ann Arbor

4. Gryntakis EM, Kim JI (1983) J Radioanal Chem 76:341

5. Mustra CO, Freitas MC, Almeida SM (2003) J Radioanal Nucl Chem 257:539

6. Hung TV, Sakamoto Y, Yasuda H (1998) Calculation of neutron flux characteristics of dalat reactor using MCNP4A code. JAERIResearch 98-057, October 1998

7. Hung TV (2010) J Radioanal Nucl Chem 283:707

8. Briesmeister JF (ed) (2000) $\mathrm{MCNP}^{\mathrm{TM}}$ - a general Monte Carlo N-particle transport code, LA 1265-M, Version 4C. Los Alamos Laboratory, Los Alamos

9. Jacimovíc R, Maucec M, Trkov (2003) J Radioanal Chem 3:513

10. Shibata K et al. (1990) Japanese evaluated nuclear data library. Version 3 -JENDL-3, JAERI 1319

11. De Corte F, Simonits A, De Wispelaere A, Hoste J (1987) J Radioanal Nucl Chem 113:145 